\title{
CCHP system operation strategy research in one of Shanghai International Tourism Resort
}

\author{
Lei Zhang, Qifen Li, Guihai Jiao, Jingjing Xu, Jialin Zhao, Junpeng Mao, \\ Yuntao Zhu, Zongqin Hou, Xiaoyu Liang \\ College of Energy and Mechanical Engineering, Shanghai University of Electric Power, China
}

Keyword: CCHP system; MATLAB; Operating optimization strategy

Abstract: This paper presents the working principle of CCHP system, and establishes the static mathematical models of Gas engine, LiBr absorption chiller, Gas boiler and Centrifugal water chiller. In this paper, it establishes optimization mathematical model that makes minimum annual total costs as optimize targets and energy balance as constraint condition; it takes the core area of a tourist resort in Shanghai as the user end, uses MATLAB software to optimize and analysis the research case, gets total annual cost and operating optimization strategy.

\section{introduction}

Due to the composition and operation of the system is more complex, and maintenance costs are also higher than the general sub supply system, if it's design or running is not reasonable, it's economy and energy efficiency can not be reflected, this requires that the energy of the CCHP system changes with the time seasons and time, different operating policies of the system will produce different energy.

\section{OPTIMIZATION MODEL OF CCHP SYSTEM}

In this paper it uses the internal combustion engine unit, waste heat unit, and lithium bromide absorption air conditioning unit, and the specific processes shown in figure 1.

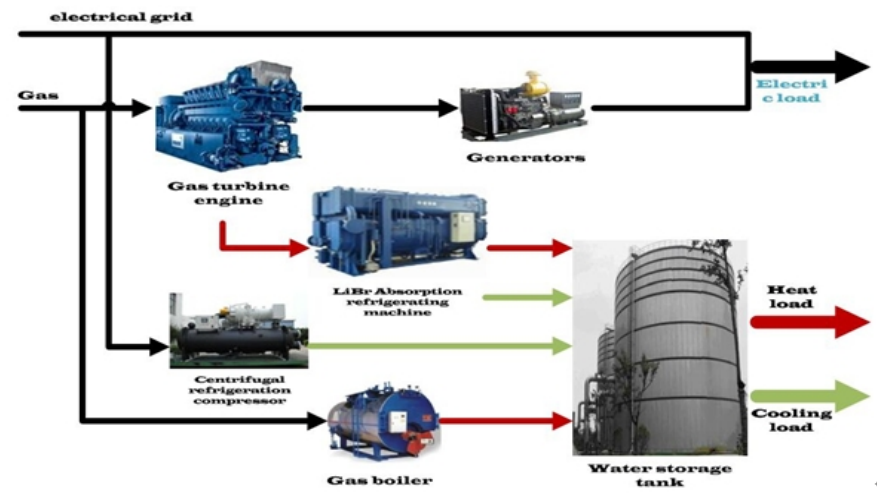

Figure 1. Gas engine fuel supply system diagram.

\section{Establishment of mathematical mode and objective function}

\section{The objective function}

This paper takes the lowest total annual cost as objective function, it can be expressed as:

$$
J=J_{C O S T}+J_{W X}+J_{O C}
$$

In the equation: $\mathbf{J}$ is the annual cost; $\mathrm{J}_{\mathrm{COST}}$ is the intial investment cost; $\mathrm{J}_{\mathrm{WX}}$ is the annual maintenance cost; $\mathrm{J}_{\mathrm{OC}}$ is the annual operating expense.

$$
J_{C O S T}=[R(1-\theta)+i \theta] *\left(J_{G E}+J_{G B}+J_{C C}+J_{S}+J_{A R}\right)
$$




$$
R=i(1+i)^{y} /\left\{(1+i)^{y}-1\right\}
$$

In the equation: $R$ is investment recovery coefficient; $\theta$ is residual rate; $i$ is annual interest rate; $y$ is Equipment life; $J_{G E}, J_{G B}, J_{C C}, J_{S}, J_{A R}$ respectively indicates initial investment cost of gas engine, gas boiler, water cooling machine, energy storage device and absorption refrigeration machine.

$$
J_{W X}=\varphi\left(J_{G E}+J_{G B}+J_{C C}+J_{S}+J_{A R}\right)
$$

In the equation: $\varphi$ is scale factor.

$$
J_{O C}=\sum_{K=1}^{K}\left[\theta_{g a s, k}\left(G_{G E, K}+G_{G B, K}\right)+\theta_{e l, k} P_{B U Y, K}\right] \Delta t_{k}(2-5)
$$

In the equation: $\theta_{g a s, k}$ and $\theta_{e l, k}$ respectively indicate the gas price points and the price of purchasing power.

\section{Mathematical model of main equipment}

In order to simplify optimization, this paper makes the following assumptions for the systems; Energy supply and demand on the user side are independent; CCHP system is 100\% reliable; CCHP system's heating and power load can change in a short time from $0 \%$ to $100 \%$.

\section{Mathematical model of gas engine}

$$
\begin{aligned}
& P_{G E, K} \leq P_{0} \\
& G_{G E, K}=G_{G E, g a s, K} * Q_{0} \\
& P_{G E, K}=G_{G E, K} \mu_{G E} \\
& Q_{G E, K}^{r e}=G_{G E, K} \mu_{R E}
\end{aligned}
$$

In the equation: $P_{G E, K}$ is the internal combustion engine's actual generating capacity; $P_{0}$ is gas engine rated power; $G_{G E, K}$ is amount of fuel for internal combustion engine unit's input; $G_{G E, g a s, k}$ is gas consumption that gas engine-generator requires; $Q^{r e}{ }_{G E, K}$ is heat supply of gas engine; $Q_{0}$ is calorific value of natural gas in the standard case; $\mu_{G E}$ and $\mu_{R E}$ respectively indicates power generation efficiency and heat transfer efficiency of internal combustion engine.

\section{The LiBr absorption refrigeration machine}

$$
\begin{aligned}
& Q_{A R, K}^{C}=Q_{A R, K}^{r e} C O P_{A R, C} \\
& Q_{A R, K}^{r}=Q_{A R, K}^{r e} C O P_{A R, r} \\
& Q_{A R, K}^{r e} \leq Q_{G E, K}^{r e}
\end{aligned}
$$

In the equation: $Q^{r e}{ }_{A R, K}$ is the $\mathrm{LiBr}$ absorption heat; $Q_{A R, K}^{r}$ is the heat load of the $\mathrm{LiBr}$ absorption chiller; $\quad Q_{A R, K}^{c}$ is the cooling load of the $\mathrm{LiBr}$ absorption chiller; $C O P_{A R, C}$ and $C O P_{A R, r}$ respectively indicates refrigeration coefficient and thermal coefficient of $\mathrm{LiBr}$ unit.

\section{Gas boiler}

$$
\begin{aligned}
& Q_{G B, K}=Q_{G B, K} \mu_{G B} \\
& G_{G B, K}=G_{G B, g a s, k} * Q_{0}
\end{aligned}
$$

In the equation: $Q_{G B, K}$ is Heat load provided by gas boiler; $G_{G B, g a s, k}$ is Gas consumption of gas boiler; $Q_{G B, K}$ is calorific value of natural gas in the standard case; $\mu_{G B}$ is boiler thermal efficiency.

\section{Centrifugal water cooling unit}

$$
Q_{C C, K}^{C}=P_{C C, K} C O P_{C C}
$$


In the equation: $Q^{C}{ }_{C C, K}$ is cooling load provided by centrifugal water chiller; $C O P_{C C}$ is Refrigeration coefficient of centrifugal water chiller.

\section{The main constraint}

Electric balance

$$
P_{G E}(K)+P_{B U Y}(K)=P^{D}(K)+P_{C C}(K)+P_{K}(K)(2-16)
$$

In the equation: $P_{B U Y}(K)$ is the purchase of electricity from the outside; $P^{D}(K)$ is power consumption required by users; $P_{C C}(K)$ is power consumption of centrifugal water chiller unit; $P_{K}(K)$ is Power consumption of the system.

\section{Gas consumption balance}

$$
G_{G T}(K)+G_{G B}(K)=G_{g a s}(K)
$$

In the equation: $G_{G T}(K)$ is the consumption of natural gas consumption of gas engine group; $G_{G B}(K)$ is natural gas consumption of gas boiler; $G_{g a s}(K)$ is total natural gas consumption.

\section{Cold load balance}

$$
Q_{A R}^{C}(K)+Q_{C C}^{C}(K)+Q_{s}(K)=Q^{D, C}(K)
$$

In the equation: $\mathrm{Q}_{\mathrm{S}}(\mathrm{K})$ is cooling capacity of accumulator, $\mathrm{Q}^{\mathrm{D}, \mathrm{C}}(\mathrm{K})$ is cold load required by users.

The above mathematical model is an optimization problem with multiple variables and constrained nonlinear functions

\section{Case analysis}

\section{Load characteristics}

Figure 2 shows hourly load characteristics of a typical daily load diagram for the summer. Due to the summer tourist resort in the daytime is relatively hot, summer cooling load and electric load at the peak of 10:00-22:00.

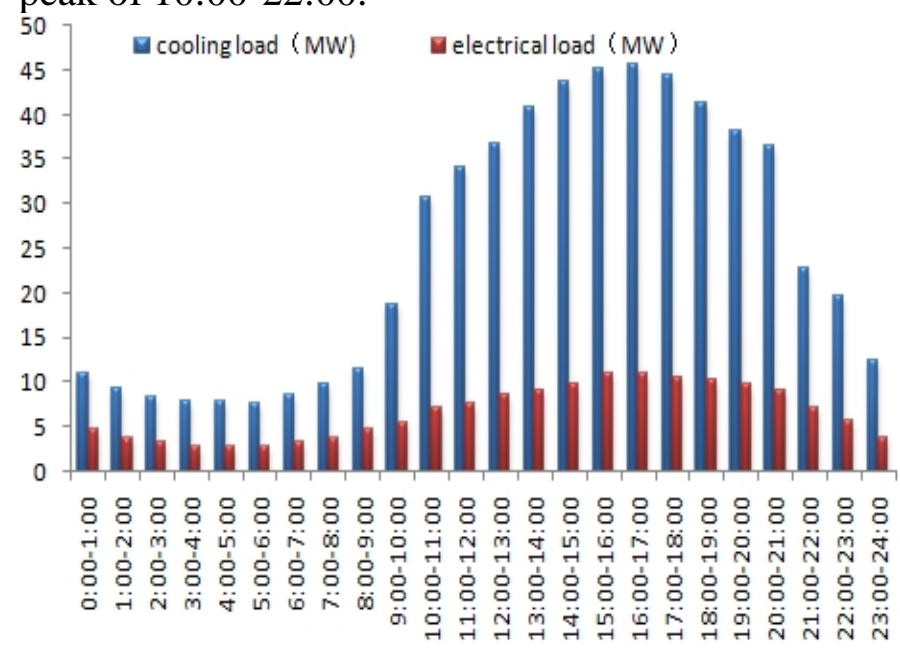

Figure 2. Cooling load and electric load of typical day in summer.

\section{Model parameters}

Energy price:

The peak price is 1.05 yuan, the peak valley electricity price of 0.56 yuan; Natural gas price is 3.05 yuan $/ \mathrm{Nm}^{3}$, the calorific value of natural gas is $35.2 \mathrm{MJ} / \mathrm{Nm}^{3}$.

Economic parameters:

$\theta=0, i=0.05, y=15, \varphi=0.03$.

Equipment parameters:

$P_{0}=4 \mathrm{MW} / \mathrm{h}, G_{G E, g a s, k}=888.4 \mathrm{NM}^{3} / \mathrm{h}, \mu_{G E}=45.4 \%, \mu_{R E}=0.9 ; Q_{A R, K}^{r}=3478 \mathrm{kw}, Q_{A R, K}^{c}=3490 \mathrm{kw}, C O P_{A R, r}=$ $0.9, C O P_{A R, C}=1.4 ; G_{G B, g a s, k}=926 \mathrm{NM}^{3} / \mathrm{h}, Q_{G B, K}=7000 \mathrm{kw}, \mu_{G B}=0.9 ; C O P_{C C}=4.5, Q^{C}{ }_{C C, K}=6238 \mathrm{kw}$. 


\section{Optimized operation result analysis}

The results are obtained by establishing the model of the cold heat and power supply system and the optimization calculation, as shown below:

$$
J=J_{C O S T}+J_{W X}+J_{O C}=115.96 \text { (million yuan) }
$$

Compared with the same situation of the sub production system, Annual operating cost of the system saves $10.87 \%$, it improves energy utilization, and The waste heat of gas engine has been fully utilized.

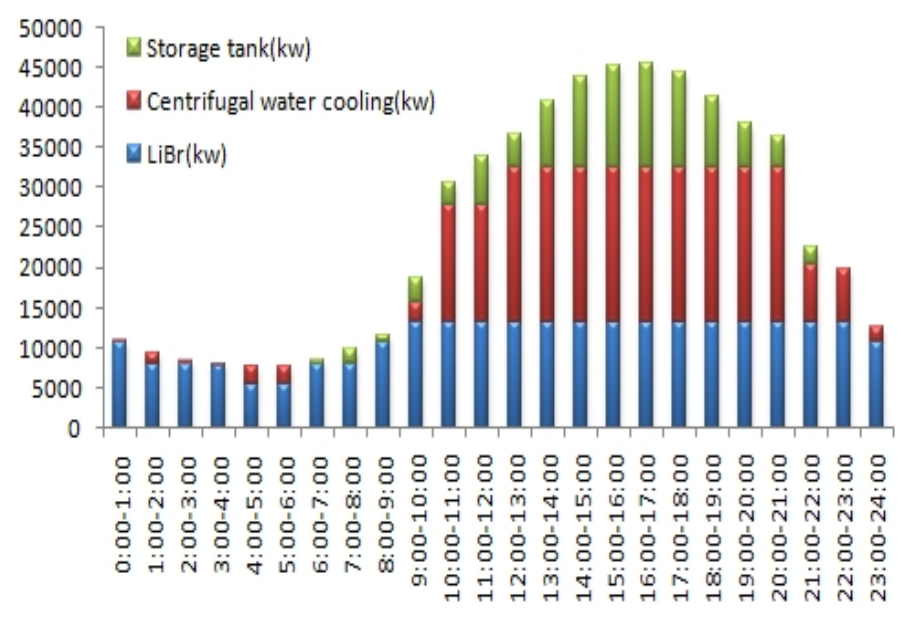

(a). Cooling load in summer.

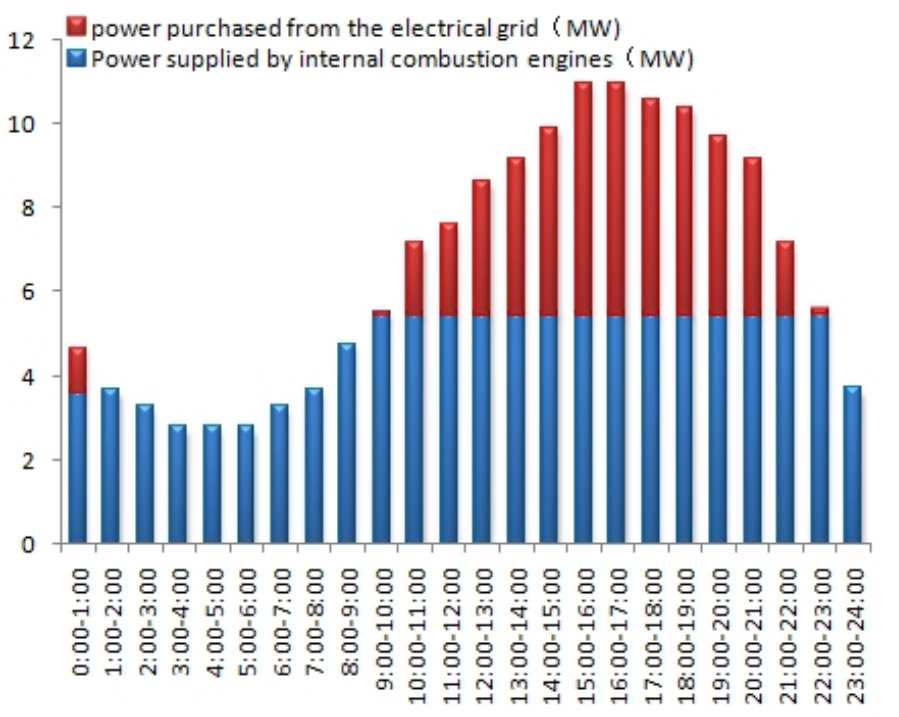

(b). Electric load in summer.

Figure 3. Optimized operation strategy diagram of the system.

As shown in figure 3(a), (b), combined with the load characteristics of the summer holiday resort, Before 8:00 o'clock in the morning, as a tourist resort area has not started, the cooling load demand is relatively small, only the lithium bromide host provide cooling capacity, excess cold storage to storage tank, and the peak load is released. After 8:00, with the increase of cooling capacity for end users, the lithium bromide operate fully load, due to the cooling capacity can not meet the needs of users, the centrifugal water cooler begins to open to supply cold quantity. At the same time, the cold storage tank will release the saving cold, it plays a role in peak shaving, alleviate the cold demand at peak. after 22:00, the users' demands for cold start to decline, it just needs the lithium bromide unit to provide cooling .but the price and the cold load are in a low state, and the water storage tank release many cold in the day. For the second day to meet the needs of users, it is reasonable to open the centrifugal water cooling unit for cooling at this time. The gas engine power generation provides 
power at idler time, In great demand for electricity, when the internal combustion engine can not meet the demand for electricity, the electricity will be purchased of electricity from the outside.

\section{The influence of natural gas prices}

The price of natural gas is one of the main factors which restrict the total cost of the whole system. To this, the state has taken various measures to encourage the development of such a high efficiency as the cold heat and power system, such as high efficiency, low pollution projects, one of the main measures is to reduce fuel costs, adjust fuel prices. Therefore, it is necessary to study the effect of natural gas prices on the economy of the whole system. This paper analyzes the economic nature of the natural gas cooling cogeneration system and the impact of natural gas price changes on the economy of the cold heat and power cogeneration system. As shown in Figure 4:

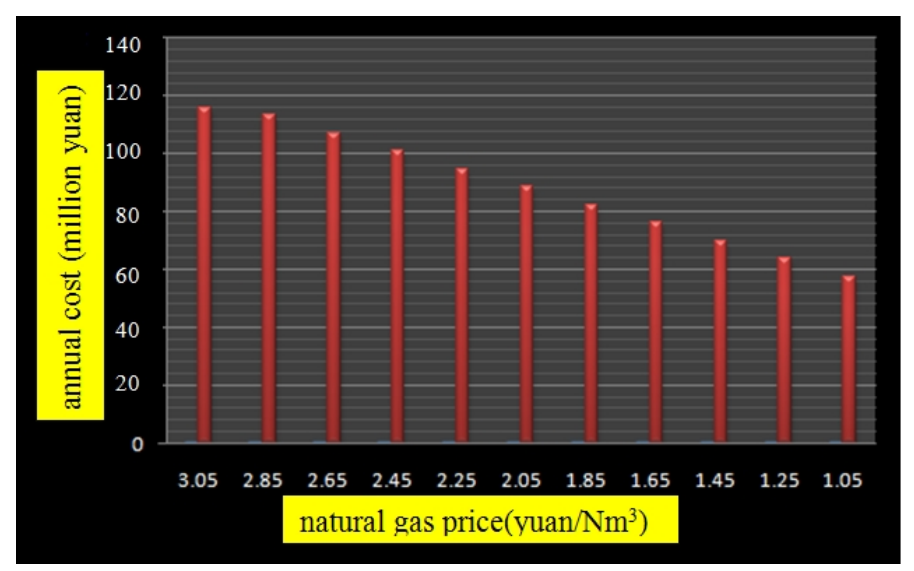

Figure4. The annual total cost of different gas prices for system

From the figure, the impact of price changes on the total cost of the system is crucial, when the gas price drops, The annual total cost will be significantly decreased.

\section{Conclusions}

By establishing optimization model for cogeneration system, Optimizing analysis of combined cooling, heating and power system of the energy station in the core area of a tourist resort in Shanghai was made. According to user demand for cooling, thermal and electrical load on typical days in summer, winter and transition seasons, the genetic algorithm of the software of MATLAB was adopted to solve the model, and therefore the minimum total annual cost was calculated and the optimal operation strategy could be worked out. From the results we can see that the optimized system operation strategy can remarkably save cost and at the same time increase energy efficiency.

\section{Reference}

[1] 2001. Standard Method of Test for the Evaluation of Building Energy Analysis Computer Programs[S]. ASHRAE Standard Project Committee 140. ANSI/ASHRAE Standard 140-2001.

[2] X.Q.Kong, R.Z.Wang, X.H.Huang. 2005. Energy optimization model for a CCHP system with available gas turbines. Applied Thermal Engineering 25:377-391.Chinese.

[3]Z.G.Ma, Y.Wen, J.Jin. Optimization of the distributed energy system. Energy Engineering 2012 (06). Chinese.

[4]S.R.Guan, J.G. Chen, J.L.Yuan. Study and optimization of natural gas distributed energy operation mode. Natural gas technology and economy 2014(01). Chinese.

[5]L.S.Cen. Distributed energy system optimization design and development mode is proposed. Application of energy 2011(02). Chinese. 